\title{
Coronal MHD transport theory and phenomenology
}

\author{
L.J. Milano*, W.H. Matthaeus*, P. Dmitruk* and S. Oughton ${ }^{\dagger}$ \\ * Bartol Research Institute, University of Delaware \\ $\dagger$ Department of Mathematics, University of Waikato
}

\begin{abstract}
In the presence of a weakly inhomogeneous background, magnetohydrodynamic fluctuations are transported, reflected and at small scales, dissipated. In contrast to orderings appropriate to outer solar wind conditions, here we explore transport in a regime relevant for solar coronal heating and solar wind acceleration, in which effects of the order of the Alfvén speed are retained while disregarding the solar wind velocity. We consider the general properties of the transport equations as well as some solutions of interest.
\end{abstract}

\section{INTRODUCTION}

MHD fluctuations in a nonuniform plasma such as the solar corona or solar wind are often studied by means of a two-scale expansion method. The best known approach, so-called "WKB theory" $[1,2]$, is valid for relatively high-frequency, non-interacting Alfvén waves. However, if the fluctuations are low frequency, or if strong mode-mode coupling (e.g., turbulence) is present, then the WKB orderings break down and a more general form of transport theory is required. Fairly general approaches for carrying out such two-scale expansion have been presented $[3,4]$ including effects associated with the large-scale (wind) flow $\mathbf{U}_{\mathbf{0}}$, and the large-scale magnetic field $\mathbf{B}_{0}$ and density $\rho$. Transport formulations of this type, adapted for outer heliospheric solar wind conditions in the super-Alfvénic wind $\left(U_{0} \gg V_{A}\right.$ where the Alfvén speed is $\left.V_{A}=B_{0} / \sqrt{4 \pi \rho}\right)$, have proven useful [5]. For these applications one typically simplifies the transport equations by assuming that $V_{A} / U_{0} \ll 1$ and dropping terms of order of this ratio. In the present paper we consider another interesting regime, one that is appropriate to the corona. Here we assume $U_{0} / V_{A} \ll 1$ and drop terms accordingly in the full transport formalism. This leads to relatively simple equations that can provide insights that complement direct calculations based upon the full primitive equations.

We begin by decomposing the magnetohydrodynamic (MHD) fields into mean and fluctuating components as follows:

$$
\begin{aligned}
\frac{\mathbf{B}}{\sqrt{4 \pi \rho}} & =\mathbf{V}_{A}(\mathbf{s}, t)+\mathbf{b}(\mathbf{s}, \mathbf{x}, t), \\
\mathbf{v} & =\mathbf{U}_{0}(\mathbf{s}, t)+\mathbf{v}(\mathbf{s}, \mathbf{x}, t),
\end{aligned}
$$

where $\rho$ is the plasma density, and $\mathbf{s}$ and $\mathbf{x}$ are formally independent variables, representing respectively slow and fast variations. The slow coordinate $\mathbf{s}$ spans large-scale distances, of the order of $1 R_{\odot}$, while the fast coordinate is associated to local turbulent dynamics. The capitalized fields are obtained via an ensemble average over the total fields $\left(\mathbf{U}_{0} \equiv\langle\mathbf{v}\rangle, \mathbf{V}_{A} \equiv\langle\mathbf{B} / \sqrt{4 \pi \rho}\rangle\right)$, and represent slowly varying background fields (respectively the Wind and Alfvén velocities). The ensemble average is assumed to filter out fast variations, and can be thought of as a volumetric average over the fast variable. Inserting Eqs. (1) in the MHD equations, one obtains [4]:

$$
\begin{gathered}
\partial_{t} \mathbf{z}^{ \pm}+\mathbf{Z}_{0}^{\mp} \cdot \nabla \mathbf{z}^{ \pm}+\mathbf{z}^{\mp} \cdot \nabla \mathbf{Z}_{0}^{ \pm} \pm R^{ \pm} \mathbf{b} \pm r^{ \pm} \mathbf{V}_{A}= \\
-\frac{1}{\rho} \nabla p+N_{0}^{ \pm}+D^{ \pm}
\end{gathered}
$$

where

$$
\begin{array}{rlrl}
\mathbf{z}^{ \pm} & \equiv \mathbf{u} \pm \mathbf{b}, & r^{ \pm}=\nabla \cdot\left(\frac{1}{2} \mathbf{u} \pm \mathbf{b}\right), \\
\mathbf{Z}_{0}^{ \pm} \equiv \mathbf{U}_{0} \pm \mathbf{V}_{A}, & R^{ \pm}=\nabla \cdot\left(\frac{1}{2} \mathbf{U}_{0} \pm \mathbf{V}_{A}\right),
\end{array}
$$

$\nabla=\nabla_{s}+\nabla_{x}$ is the total gradient operator, $p$ is the total (kinetic plus magnetic) pressure, $D^{ \pm}$represents the dissipation terms, and $N_{0}^{ \pm}$the nonlinear terms. The last term in the LHS of Eq. (2) was not present in Eq. (21) of Ref. [4], where terms of order $V_{A}$ were neglected with respect to terms of order $U_{0}$, which is a valid approximation at distances $\geq 1 \mathrm{AU}$. Here we retain terms of order $V_{A}$, and we assume local incompressibility, as well as homogeneity and isotropy in the fast variable. Fine points of the general derivation are discussed in Ref. [4].

December 18, 2003 343 


\section{TRANSPORT IN RMHD}

We further simplify the treatment by postulating a rectangular geometry in which $\mathbf{s}$ is orthogonal to $\mathbf{x}$ :

$$
\mathbf{x}=(x, y, 0), \quad \nabla_{x}=\left(\partial_{x}, \partial_{y}, 0\right), \quad \mathbf{z}^{ \pm}=\left(z_{x}^{ \pm}, z_{y}^{ \pm}, 0\right),
$$

$\mathbf{s}=(0,0, s), \quad \nabla_{s}=\left(0,0, \partial_{s}\right), \quad \mathbf{Z}_{0}^{ \pm}=\left(0,0, U_{0} \pm V_{A}\right)$.

Under this approximation, expansion and curvature effects are neglected, thus reducing mathematical complexity. We postpone investigation of these higher-order corrections to future efforts.

In rectangular geometry, the scale-separated MHD equations Eq. (2) take the reduced (hence the name Reduced MHD, or RMHD) form:

$\partial_{t} \mathbf{z}^{ \pm}+\left(U_{0} \mp V_{A}\right) \partial_{s} \mathbf{z}^{ \pm} \pm \frac{1}{2} R^{ \pm}\left(\mathbf{z}^{+}-\mathbf{z}^{-}\right)=N_{0}^{ \pm}+D^{ \pm}$,

where we explicitly wrote $\mathbf{b}=\frac{1}{2}\left(\mathbf{z}^{+}-\mathbf{z}^{-}\right)$. We define the usual correlation tensors [4]:

$$
\begin{aligned}
& H_{i j}^{ \pm} \equiv\left\langle z_{i}^{ \pm} z_{j}^{ \pm^{\prime}}\right\rangle, \\
& \Lambda_{i j} \equiv\left\langle z_{i}^{+} z_{j}^{{ }^{\prime}}\right\rangle,
\end{aligned}
$$

where the average is over the fast variable $\mathbf{x}$, and the symbol' denotes evaluation at the displaced position $\mathbf{x}+\mathbf{r}$; i.e., if $\mathbf{z}^{ \pm}=\mathbf{z}^{ \pm}(x, y, s, t)$ then $\mathbf{z}^{ \pm^{\prime}}=$ $\mathbf{z}^{ \pm}\left(x+r_{x}, y+r_{y}, s, t\right)$.

The evolution equations for the tensors $H_{i j}^{ \pm}$and $\Lambda_{i j}$ are obtained by multiplying Eqs. (5) by $z_{i, j}^{ \pm^{\prime}}$ and then averaging. While the LHS of Eqs. (5) give a fairly straightforward contribution, which describes the linear transport of these quantities, the RHS needs to be modeled phenomenologically, as we discuss in the following sections.

\section{LINEAR ALFVÉNIC TRANSPORT}

Proceeding as explained above, one obtains:

$$
\begin{gathered}
\partial_{t} H_{i j}^{ \pm}+L_{W K B}^{ \pm} H_{i j}^{ \pm}-R^{ \pm} \Lambda_{i j}=0, \\
\partial_{t} \Lambda_{i j}+\left(U_{0} \partial_{s}+\frac{\partial_{s} U_{0}}{2}\right) \Lambda_{i j}-\frac{R^{-} H_{i j}^{+}-R^{+} H_{i j}^{-}}{2} \\
+V_{A} M_{i j}=0,
\end{gathered}
$$

where

$$
\begin{aligned}
& L_{W K B}^{ \pm} \equiv\left(U_{0} \mp V_{A}\right) \partial_{s}+R^{ \pm}, \\
& M_{i j} \equiv\left\langle z_{i}^{+} \partial_{s} z_{j}^{-^{\prime}}-z_{i}^{{ }^{\prime}} \partial_{s} z_{j}^{+}\right\rangle .
\end{aligned}
$$

The equation for $\Lambda_{i j}$ involves a new tensor $M_{i j}$, whose evolution should be computed independently in a complete treatment. However, the equation for $\partial_{t} M_{i j}$ involves not just $D_{i j}$ and $\Lambda_{i j}$, but additional tensors, with higher-order derivatives in $s$. This is a familiar type of closure problem. Our approach herein will involve modeling of $M_{i j}$.

By Alfvénic transport we mean that terms of $O\left(U_{0} / V_{A}\right)$ are neglected. In this particular case the equations reduce to:

$$
\begin{aligned}
\partial_{t} H_{i j}^{ \pm} \mp L_{W K B} H_{i j}^{ \pm} \mp R \Lambda_{i j} & =0, \\
\partial_{t} \Lambda_{i j}+\frac{1}{2} R\left(H_{i j}^{+}-H_{i j}^{-}\right)+V_{A} M_{i j} & =0,
\end{aligned}
$$

where

$$
L_{W K B} \equiv-V_{A} \partial_{s}+R \quad \text { and } \quad R \equiv \partial_{s} V_{A} .
$$

Defining the energies $E^{ \pm} \equiv\left\langle\left|\mathbf{z}^{ \pm}\right|^{2}\right\rangle=\left.H_{i i}^{ \pm}\right|_{r=0}$, and energy difference $D^{ \pm} \equiv\left\langle\mathbf{z}^{+} \cdot \mathbf{z}^{-}\right\rangle=\left\langle|\mathbf{u}|^{2}-|\mathbf{b}|^{2}\right\rangle=$ $\left.\Lambda_{i i}\right|_{r=0}$, one readily obtains,

$$
\begin{gathered}
\partial_{t} E^{ \pm} \mp L_{W K B} E^{ \pm} \mp R D=0, \\
\partial_{t} D+\frac{1}{2} R\left(E^{+}-E^{-}\right)+V_{A} M=0,
\end{gathered}
$$

where $\left.M \equiv M_{i i}\right|_{r=0}=\left\langle\mathbf{z}^{+} \cdot \partial_{s} \mathbf{z}^{-}-\mathbf{z}^{-} \cdot \partial_{s} \mathbf{z}^{+}\right\rangle$. Note that a conveniently defined cross helicity is $H_{c} \equiv$ $4\langle\mathbf{u} \cdot \mathbf{b}\rangle=E^{+}-E^{-}$.

As a first case, we look for steady solutions of Eqs. (15-16). For this case Eq. (16) shows that $V_{A} M=-\frac{1}{2} R H_{c}$. Subtracting Eqs. (15) we obtain:

$$
L_{W K B} H_{c}=0 \quad \Rightarrow \quad H_{c}(s)=a V_{A}(s),
$$

where $a$ is a constant to be determined by the boundary conditions. It follows that the steady state expression for $M$ is given by:

$$
M(s)=-\frac{a}{2} R(s)
$$

These steady linear solutions are exact. However, we still cannot integrate Eqs. (15) to obtain $E^{+}$and $E^{-}$, since in steady state Eq. (16) does not provide any information on $D$. Note that $M$ is proportional to the reflection coefficient, and thus tends to zero when $\partial_{s} V_{A} \rightarrow 0$. In fact, in the absence of reflections the steady solution is trivial: both $E^{+}$and $E^{-}$are constant in $s$, and the energy difference does not play any significant role in the (linear) dynamics.

As a second example, we can gain insight into the role of the energy difference $D$ by looking at the early evolution starting from the initial condition: $E^{-}=E_{0}^{-}=$const, $E^{+}=0 \Rightarrow D=0$. For short times 
$\left(t \ll 1 / R_{\max }\right)$, we assume that $\partial_{s} \mathbf{z}^{ \pm}=0$ and drop all $\partial_{s}$ terms in Eqs. (15-16), including $M$. We obtain:

$$
\begin{array}{r}
E^{+}(t)=E_{0}^{-} \frac{1}{4} R^{2} t^{2}, \\
E^{-}(t)=E_{0}^{-}\left(1-\frac{1}{4} R^{2} t^{2}\right), \\
D(t)=E_{0}^{-} \frac{1}{2} R t .
\end{array}
$$

Note that $D$ grows linearly in time and helps transfer energy from the dominant field ( $z^{-}$in this example) to the weaker field $\left(z^{+}\right)$This linear growth will saturate when $M$ balances the cross helicity contribution in Eq. (16).

We now consider a case in which the system of equations (15)-(16) can be closed. This is the case in which only one of the fields, say $z^{-}$, is injected at a low frequency $\left(\omega_{0} \ll \omega_{A}\right)$ from the boundaries, and the reflections are weak $\left(R \ll \omega_{A}\right)$, where $\omega_{A}$ is the Alfvén frequency $\omega_{A} \equiv 2 \pi / t_{A}$, with the Alfvén transit time $t_{A} \equiv L_{s} /\left\langle V_{A}\right\rangle_{s}$ defined in terms of the $s$ averaged Alfvén velocity $\left\langle V_{A}\right\rangle_{s}$ and the length of the system in the slow direction $L_{s}$, which is typically about $1 R_{\odot}$. In this case, we expect the system to be close to the zero-order solution (the solution for $R=0): \mathbf{z}_{(0)}^{+}=0$, and $\mathbf{z}_{(0)}^{-}$a solution to the wave equation $\partial_{t} \mathbf{z}_{(0)}^{-}=-V_{A} \partial_{s} \mathbf{z}_{(0)}^{-}$. That is,

$$
\begin{array}{r}
\mathbf{z}^{+}=\mathbf{z}_{(1)}^{+}, \\
\mathbf{z}^{-}=\mathbf{z}_{(0)}^{-}+\mathbf{z}_{(1)}^{-}, \\
z_{(1)}^{+} \sim z_{(1)}^{-} \ll z_{(0)}^{-},
\end{array}
$$

where $z^{ \pm} \equiv\left\langle\left|\mathbf{z}^{ \pm}\right|^{2}\right\rangle^{1 / 2}$. We will show that under these conditions we can write

$$
M=-\partial_{s} D+\delta M
$$

where $\delta M \equiv M+\partial_{s} D$ is a small correction that tends to zero when both $\omega_{0}$ and $R$ tend to zero. This relationship (evaluated at $\delta M=0$ ) allows a closed system of equations (15)-(16) for $E^{+}, E^{-}$and $D$.

We first note that $M$ can be rewritten as:

$$
M=-\partial_{s} D+2\left\langle\mathbf{z}^{+} \cdot \partial_{s} \mathbf{z}^{-}\right\rangle,
$$

where we used the product rule on $\partial_{s}\left(\mathbf{z}^{+} \cdot \mathbf{z}^{-}\right)$and the definition of $D=\left\langle\mathbf{z}^{+} \cdot \mathbf{z}^{-}\right\rangle$. Under the assumed conditions, the second term on the RHS of Eq. (22) is negligible. To show this, we estimate the $s$ derivatives of the fields. While the zero-order solution is driven by the boundary condition at frequency $\omega_{0}$, the perturbed component oscillates at the much faster Alfvén frequency $\omega_{A}$ :

$$
\begin{aligned}
& V_{A} \partial_{s} z_{(0)}^{-} \sim \omega_{0} z_{(0)}^{-}, \\
& V_{A} \partial_{s} z_{(1)}^{ \pm} \sim \omega_{A} z_{(1)}^{ \pm} .
\end{aligned}
$$

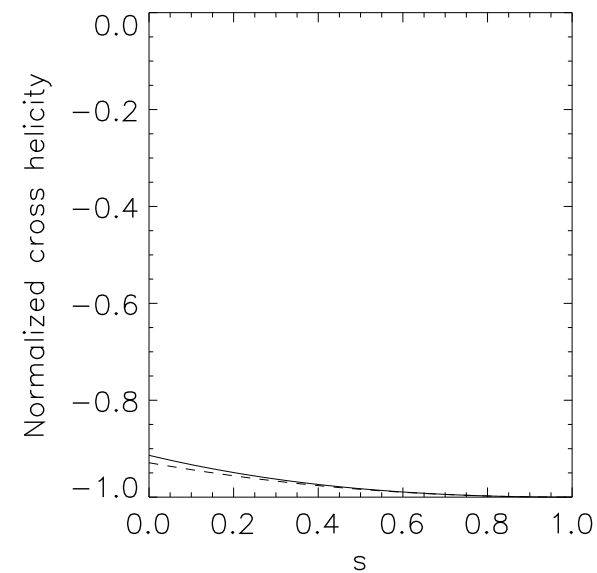

FIGURE 1. Time averaged linear solution of the MHD equations (dashed line), compared with the (linearized) closure equations $(3-4,10)$. The Alfvén profile is linear, with $R L_{s} / V_{A}=0.5$ and $w_{0} / w_{A}=0.1$.

These estimates imply $V_{A} \partial_{s} D \sim \omega_{A} z_{(0)}^{-} z_{(1)}^{+}$, and thus,

$$
\frac{\left\langle\mathbf{z}^{+} \cdot \partial_{s} \mathbf{z}^{-}\right\rangle}{\partial_{s} D} \sim \frac{\omega_{0}}{\omega_{A}}+\frac{z_{(1)}^{-}}{z_{(0)}^{-}} \ll 1,
$$

as we anticipated above.

We test this approximate closure and show a diagnostic plot in Fig. 1. The figure compares the normalized cross helicity $\left[\sigma=\left(E^{+}-E^{-}\right) /\left(E^{+}+E^{-}\right)\right]$ as computed: (i) exactly, from the integration of the linearized MHD equations for a linear Alfvén profile, and (ii) from our closure linear equations. We use a linear Alfvén profile, featuring moderate reflections $\left(R=1 / 2 \omega_{A}\right)$. The boundary conditions are: no incoming waves $\left[z^{+}\left(s=L_{s}\right)=0\right]$, and low-frequency $\left(\omega_{0}=\omega_{A} / 10\right)$ outgoing $\left(z^{+}\right)$waves injected from the photosphere (at $s=0$ ). The exact solution is obtained from the RMHD equations for a single perpendicular mode, as in [6] (linearized Eqs. (10-12) from Ref. [6]). Since the periodicity in the boundary condition introduces a time periodicity in the solutions, we time-average over one period $\left(2 \pi / \omega_{0}\right)$ and make use of the steady state solutions. The comparison shows fairly good agreement. Flatter Alfvén profiles give better agreement, as expected.

\section{NON-LINEAR TRANSPORT}

Anticipating that the solar corona may be in a turbulent state, we briefly discuss a strategy to handle MHD non-linearities in the transport equations.

December 18, 2003 
So far we have neglected the nonlinear and dissipative terms (respectively $N_{0}^{ \pm}$and $D^{ \pm}$) in the RHS of Eq. (5). It is well known that the non-linear terms transport directly cascading ideal invariants (such as $E^{ \pm}$) through intermediate (inertial range) scales, toward small scales where they are dissipated. Even though the problem seems conceptually simple, the mathematical description is extremely difficult, with no complete rigorous theory of the process being known. For a macroscopic description of the problem where the focus is in the transport and dissipation of the fluctuations, an effective way to handle it consists of two main steps: (1) neglect dissipative terms, an excellent approximation at large scales; (2) replace the exact non-linear terms by phenomenologically modeled non-linear terms. The second step is the more difficult. One example of such a phenomenology is given by the following model [7]:

$$
\partial_{t} E_{N}^{ \pm}=-\alpha^{ \pm} \frac{\sqrt{E^{\mp}}}{\lambda^{ \pm}} E^{ \pm}
$$

where $\partial_{t} E_{N}^{ \pm}$stands for the non-linear contribution to $\partial_{t} E^{ \pm}$, which has to be added to the RHS of Eqs. (15). Here $\alpha^{ \pm} \sim 1$ and $\lambda^{ \pm}$are the correlation lengths for $E^{ \pm}$, that is,

$$
\lambda^{ \pm} \equiv \frac{\int_{0}^{\infty} H_{i i}^{ \pm} d r}{\left.H_{i i}\right|_{r=0}} \equiv \frac{L^{ \pm}}{E^{ \pm}} .
$$

It turns out that the time evolution and space distribution of $\lambda^{ \pm}(s, t)$ is not necessarily trivial. In order to show the complexity of the problem, we work with the linearized Eqs. (8) to obtain:

$$
\partial_{t} L^{ \pm} \mp L_{W K B} L^{ \pm} \mp R L^{D}=0,
$$

and assuming that $E^{+}>0$ and $E^{-}>0$,

$$
\partial_{t} \lambda^{ \pm} \mp V_{A} \partial_{s} \lambda^{ \pm} \mp R \frac{D}{E^{ \pm}}\left(\lambda^{D}-\lambda^{ \pm}\right)=0,
$$

where $L^{D}$ and $\lambda^{D}$ follow from definitions analogous to those for $L^{ \pm}$and $\lambda^{ \pm}$. Note that the linear equation (28) admits a steady solution $\lambda^{+}=\lambda^{-}=\lambda^{D}=$ constant $\equiv \lambda_{0}$. The use of this simple solution, i.e. the choice of one single similarity length characterizing all the turbulent correlations, together with Eqs. (15), (16), (21) and (25), gives a complete set of equations for the energies and dissipation of the outgoing and incoming fluctuations.

\section{CONCLUSIONS}

We have proposed a model for transport of MHD fluctuations in the solar corona, through the study of two-point correlation functions. The model extends previous work [4] to include a coronal scenario, for which the Alfvén velocity is much greater than the wind speed. Reflections, derived from the inhomogeneity of the large-scale Alfvén velocity, play an essential role in redistributing energy between incoming and outgoing fluctuations. Thus, an initial configuration with only outgoing waves would, after a transient period of time of about $1 / R$ (where $R$ is the reflection coefficient), reaches a state in which both outgoing and incoming fluctuations coexist, allowing for non-linear interactions and turbulence.

The system of equations for the time evolution of two-point correlations suffers a closure problem. However, this problem can be overcome in the asymptotic limit in which reflections are smooth, and only one type of fluctuation (either outgoing or incoming) is injected at a low frequency. In this case, an approximate closure can be used and a closed system of equations obtained.

We also briefly discussed a strategy to deal with non-linearities in the model equations. However, a better understanding of the phenomenology of MHD turbulence is required for a more accurate modeling of the non-linear terms in the transport equations.

\section{ACKNOWLEDGMENTS}

We acknowledge support by the NSF (ATM 0105254), NASA (NAG5-8134), and UK PPARC (PPA/G/S/1999/00059). This research has made use of NASA's Astrophysics Data System.

\section{REFERENCES}

1. Parker, E. N., ApJ, 143, 32-+ (1966).

2. Hollweg, J. V., J. Geophys. Res., 78, 3643-3652 (1973).

3. Marsch, E., and Tu, C.-Y., Journal of Plasma Physics, 41, 479-491 (1989).

4. Zhou, Y., and Matthaeus, W. H., J. Geophys. Res., 95, 10291-10311 (1990).

5. Smith, C. W., Matthaeus, W. H., Zank, G. P., Ness, N. F., Oughton, S., and Richardson, J. D., J. Geophys. Res., 106, 8253-8272 (2001).

6. Dmitruk, P., Milano, L. J., and Matthaeus, W. H., ApJ, 548, 482-491 (2001).

7. Hossain, M., Gray, P. C., Pontius, D. H., Matthaeus, W. H., and Oughton, S., Physics of Fluids, 7, 2886-2904 (1995). 